\title{
Transcriptome analysis of the venom gland of the Mexican scorpion Hadrurus gertschi (Arachnida: Scorpiones) Elisabeth F Schwartz ${ }^{1,2}$, Elia Diego-Garcia1, Ricardo C Rodríguez de la Vega ${ }^{1}$ and Lourival D Possani*1
}

Address: ${ }^{1}$ Departamento de Medicina Molecular y Bioprocesos, Instituto de Biotecnología, Universidad Nacional Autónoma de México, Avenida Universidad, 2001 Cuernavaca 62210, Mexico and 2Departamento de Ciências Fisiológicas, Instituto de Ciências Biológicas, Universidade de Brasília, Brasília, DF, 70910-900, Brasil

Email: Elisabeth F Schwartz - efschwa@unb.br; Elia Diego-Garcia - elia@ibt.unam.mx; Ricardo C Rodríguez de la Vega - delavega@ibt.unam.mx; Lourival D Possani* - possani@ibt.unam.mx

* Corresponding author

Published: 16 May 2007

BMC Genomics 2007, 8:119 doi:10.1186/147|-2164-8-119
Received: 17 March 2007

Accepted: 16 May 2007

This article is available from: http://www.biomedcentral.com/I47I-2164/8/119

(c) 2007 Schwartz et al; licensee BioMed Central Ltd.

This is an Open Access article distributed under the terms of the Creative Commons Attribution License (http://creativecommons.org/licenses/by/2.0), which permits unrestricted use, distribution, and reproduction in any medium, provided the original work is properly cited.

\begin{abstract}
Background: Scorpions like other venomous animals posses a highly specialized organ that produces, secretes and disposes the venom components. In these animals, the last postabdominal segment, named telson, contains a pair of venomous glands connected to the stinger. The isolation of numerous scorpion toxins, along with cDNA-based gene cloning and, more recently, proteomic analyses have provided us with a large collection of venom components sequences. However, all of them are secreted, or at least are predicted to be secretable gene products. Therefore very little is known about the cellular processes that normally take place inside the glands for production of the venom mixture. To gain insights into the scorpion venom gland biology, we have decided to perform a transcriptomic analysis by constructing a cDNA library and conducting a random sequencing screening of the transcripts.
\end{abstract}

Results: From the cDNA library prepared from a single venom gland of the scorpion Hadrurus gertschi, 160 expressed sequence tags (ESTs) were analyzed. These transcripts were further clustered into 68 unique sequences ( 20 contigs and 48 singlets), with an average length of 919 bp. Half of the ESTs can be confidentially assigned as homologues of annotated gene products. Annotation of these ESTs, with the aid of Gene Ontology terms and homology to eukaryotic orthologous groups, reveals some cellular processes important for venom gland function; including high protein synthesis, tuned posttranslational processing and trafficking. Nonetheless, the main group of the identified gene products includes ESTs similar to known scorpion toxins or other previously characterized scorpion venom components, which account for nearly $60 \%$ of the identified proteins.

Conclusion: To the best of our knowledge this report contains the first transcriptome analysis of genes transcribed by the venomous gland of a scorpion. The data were obtained for the species Hadrurus gertschi, belonging to the family Caraboctonidae. One hundred and sixty ESTs were analyzed, showing enrichment in genes that encode for products similar to known venom components, but also provides the first sketch of cellular components, molecular functions, biological processes and some unique sequences of the scorpion venom gland. 


\section{Background}

Scorpion venoms are very complex mixtures with hundreds of different components produced by the highly specialized venom glands. The most prominent components of scorpion venoms are the peptides responsible for the neurotoxic effects associated with their sting, for which more than 350 different have been described (extensive databases can be found in Tox-Prot [1] and SCORPION [2]). Most of these toxins are structurally related disulphide-rich short proteins (23-75 amino acid residues long), which affect cellular communication by modulating $\mathrm{Na}^{+}$or $\mathrm{K}^{+}$ion-channels permeability [3]. Due to their importance in scorpion envenomation and their usefulness as molecular and pharmacological probes for studying ion-channels, most of the work performed to date are focused at these neurotoxins, with relative few other components ever described; among which are heterodimeric phospholipases A2 (v.gr. [4-6]), non-disulphide short peptides with cytolytic activity and a few other functions [7,8]. Recent proteomic analyses [9-16] have documented the overall composition for nine scorpion species, all of them from the family Buthidae and most of them belonging to the Tityus genus. These analyses confirmed the gross estimation of an average of one hundred different proteins in each one of the venoms [17]. Approximately half of them comprehend components with molecular masses in the range of commonly found scorpion toxins $(2,000-8,000 \mathrm{Da})$. These numbers contrast heavily with the known universe of protein components (near four hundreds) described to exist in scorpion venoms, from which only about $12 \%$ are not classified within the known scorpion toxin families.

Further insights into scorpion venom compositions have been achieved by gene cloning by PCR-based methods conducted with cDNA libraries. For example, almost one hundred toxin precursors have been sequenced from venom gland libraries of the buthid scorpion Mesobuthus martensii (v.gr. [18-20]). Unfortunately the spectrum of sequences obtained through PCR-based approach is limited by the specificity of the PCR primers used. It is worth noticing that although PCR-based methods along with the abundant isolation and characterization of scorpion toxins and, more recently, proteomic profiling of whole venoms, have provided us with a large number of sequences, all these components are secreted from the venom glands. Little is known about the biological processes that are taking place inside the venom gland cells. Therefore, we elected to use a transcriptome approach to improve the understanding of the composition of Hadrurus gertschi venom gland.

The scorpion H. gertschi Soleglad (1976) belongs to the family Caraboctonidae [21] and is considered no dangerous to humans. H. gertschi is endemic to Mexico, occurring exclusively in the State of Guerrero, and lives underground in tunnels excavated in the soil. From the venom of this scorpion few components have been isolated and studied: hadrurin, an antimicrobial and cytolytic peptide [22]; HgeTx1, a $\mathrm{K}^{+}$channel blocker [23]; hadrucalcine, a peptide capable of activating skeletal Ryanodine receptors [Schwartz et al., in preparation], and; the precursors HgeScplp and HgeßKTx, which encode for long-chain peptides similar to Scorpine and $\beta$ KTx's, respectively [24]. Although hadrurin was reported as component of $H$. aztecus venom [22], the specimens used in that work were not taxonomically identified and latter it was realized that scorpions from that geographical region should be named $H$. gertschi; this species assignment was confirmed by identification of relevant taxonomic keys in the specimens.

In the present work we randomly generated and analyzed 160 expressed sequence tags (ESTs) from a cDNA library of the venom gland of $H$. gertschi. These 160 ESTs corresponded to $0.15 \%$ of the whole non-amplified cDNA library and were generated from a non-normalized cDNA library. After clustering the resulting dataset, we identified transcripts possibly associated with different cellular functions. The possible roles of some of the transcripts are discussed, although many have unknown functions. Furthermore, we present 8 full length sequences of new toxins.

Single-pass gene sequencing from cDNA libraries is an affordable strategy to mine the transcript profile of a given tissue [25]. This strategy has been used to analyze the transcripts profiles for few other venomous organisms, such as cnidarians [26,27], cone snails [28], fishes [29], snakes (v.gr. [30-32]) and spiders [33]. To the best of our knowledge, this is the first report of an ESTs strategy conducted with any scorpion venom gland. Moreover, this is the first comprehensive molecular study of a non-buthid scorpion, which could serve for comparative purposes when studying the details of the process by which buthid scorpions have been assembling their neurotoxic arsenal.

\section{Results \\ cDNA library and EST analysis}

The $H$. gertschi venom gland library constructed was not amplified $\left(2.8 \times 10^{5} \mathrm{cfu} / \mathrm{mL}\right.$ with $99 \%$ recombinant clones); therefore the cluster size might reflect the relative abundance of the corresponding mRNA population (see [34,35], but also [36,37]). After sequencing, 160 electropherograms were submitted to bioinformatics analysis to remove vector and poor quality sequences, resulting in 147 high-quality ESTs which were used to analyze gene expression profile in the $H$. gertschi venom glands. The mean read length of ESTs was 919 nucleotides (ranging from 225 to 1613 nucleotides, Figure 1). After clusterization 20 clusters showing more than one EST and 48 singlets were grouped (Table 1). Among the 147 ESTs, in 
29\% (4 contigs and 19 singlets) we were unable to identify any open reading frame (ORF). The remaining sequences encode for protein precursors with an average of 131 residues (from 66 to 285 amino acid residues long). The complete dbEST submission with 68 nucleotide sequences and annotations is included in Additional file 2 as raw text.

\section{Similarity searches and sequence annotation}

All sequences were submitted for blastn and blastx searches against nr database; an e-value $<10^{-5}$ was used as cut-off for confidential homologue detection. In addition to the ESTs for which no clear ORF have been identified, nearly $19 \%$ of the new sequences (in 3 contigs and 10 singlets) do not match with any entry in the database. Altogether, unassigned ESTs account for close to $48 \%$ of the total dataset, a value similar to other transcriptome studies which show values varying from $13 \%$ to $56 \%$ of non matched sequences [26-33,35]. Noteworthy, these putative gene products signify a source of new information about scorpion venom gland specific genes. In addition to ESTs with no database match, 2 reads presented identity with sequences that have been already described but with no functional assessment, hereby named unknown proteins. One of these, HGE032|2273, is the clone which codes for a protein similar to the CG9896-like proteins identified in the scorpions Mesobuthus gibbosus and $M$. cyprius. The other one, HGE033|2208, encodes for a scorpion homologue of short proteins conserved in eukaryotic organisms (KOG4604, pfam04418.6), but whose function is not known yet.

The identified putative proteins (50\% of the total) were assorted into two main groups (Table 1 and Figure 2): 1) precursors similar to gene products implicated in common cellular processes account for $18 \%$ of transcripts (in 5 contigs and 15 singlets) and; 2) putative toxins or other venom components, representing $31 \%$ of total ESTs (in 8 contigs and 2 singlets). For most of these sequences, the putative identity deposited into the dbEST correspond to the eukaryotic orthologous group (KOG [38]) each would belong (see Materials and Methods), with relevant Gene Ontology (GO [39]) terms assigned with the aid of AmiGO and GOblet [40] web servers.

Table I: Identification of the transcripts predicted to be involved in common cellular processes and those similar to known venom components. The putative identity corresponds to the eukaryotic orthologous group (KOG), as detailed in Methods.

\begin{tabular}{|c|c|c|}
\hline Sequence Id & GenBank & Descriptor \\
\hline \multicolumn{3}{|c|}{ Gene products predicted to be involved in common cellular processes } \\
\hline HGE00I|Contig I 3 & EL698878 & KOGI376 Alpha tubulin \\
\hline HGE003|Contig2I & EL698880 & KOG3412 60S ribosomal protein L28 \\
\hline HGE004|Contig 12 & EL698881 & KOG0279 G protein beta subunit-like protein \\
\hline HGE005|Contig 19 & EL698882 & KOGI954 Endocytosis/signaling protein EHDI \\
\hline HGE006|2203 & EL698883 & KOG34I8 60S ribosomal protein L27 \\
\hline HGE007|2225 & EL698884 & KOG3449 60S acidic ribosomal protein P2 \\
\hline HGE008|2233 & EL698944 & KOG07I4 Molecular chaperone (DnaJ superfamily) \\
\hline HGE009|2404 & EL698885 & KOG3458 NADH:ubiquinone oxidoreductase, NDUFA8/PGIV/I 9 kDa subunit \\
\hline HGEOIO|2258 & EL698886 & KOG0863 20S proteasome, regulatory subunit alpha type PSMAI/PRE5 \\
\hline HGEOI I|2268 & EL698887 & KOG33।I Ribosomal protein SI8 (40S) \\
\hline HGEOI $2 \mid 2330$ & EL698888 & KOG I629 Bax-mediated apoptosis inhibitor TEGT/BI-I \\
\hline HGEOI $3 \mid 2397$ & EL698889 & KOG0898 40S ribosomal protein SI5 \\
\hline HGEOI4|2453 & EL698890 & KOG2597 Predicted aminopeptidase of the MI7 family \\
\hline HGEOI5|22I7 & EL698891 & KOG3752 Ribonuclease $\mathrm{H}$ \\
\hline HGEOI $7 \mid 2209$ & EL698945 & KOG0876 Manganese superoxide dismutase \\
\hline HGEOI8|2232 & EL698892 & KOG294I Beta-I,4-mannosyltransferase \\
\hline HGE020|2328 & EL698894 & KOG4075 Cytochrome c oxidase, subunit IV/COX5b \\
\hline HGE02I|2448 & EL698895 & KOG2667 COPII vesicle protein \\
\hline HGE022|contig I 7 & EL698896 & KOG2403 Succinate dehydrogenase, flavoprotein subunit \\
\hline HGE023|2323 & EL698897 & KOG2486 Predicted GTPase \\
\hline HGE033|2208 & $\underline{E L 698907}$ & KOG4604 Uncharacterized conserved protein \\
\hline \multicolumn{3}{|c|}{ Gene products similar to known venom components } \\
\hline HGE024|Contig2 & EL698898 & $\alpha-K T \times 6$ subfamily \\
\hline HGE025|Contig5 & EL698899 & Novel $\alpha-\mathrm{KTx}$ \\
\hline HGE034|Hgscplike I & EL698908 & Scorpine-like group \\
\hline HGE026|Hgscplike2 & EL698900 & Scorpine-like group \\
\hline HGE035|HgbetaKTxI & $\underline{E L 698909}$ & Novel $\beta-\mathrm{KTx}$ \\
\hline HGE027|NDPB_5.5 & EL698901 & Novel NDBP group 5 \\
\hline HGE028|NDPB_5.6 & EL698902 & Novel NDBP group 5 \\
\hline HGE029|NDPB_3.7 & EL698903 & Novel NDBP group 3 \\
\hline HGE03I|PLA2 & $\underline{E L 698905}$ & Novel group III heterodimeric phospholipase \\
\hline HGE030|HgI & EL698904 & KOG4295 Serine proteinase inhibitor (KU family) \\
\hline
\end{tabular}




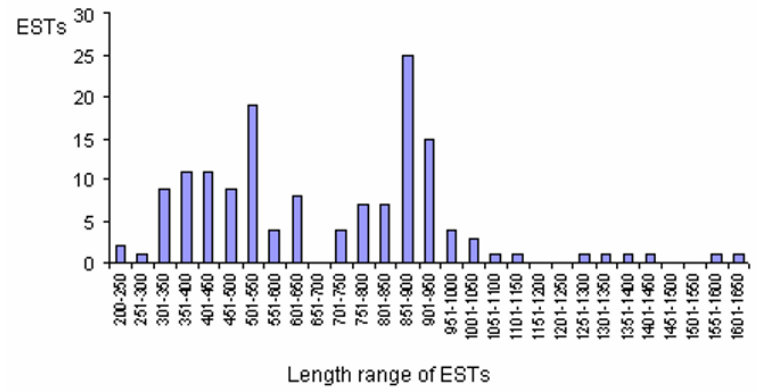

Figure I

Reads length distribution of $\boldsymbol{H}$. gertschi venom gland ESTs. A total of I47 ESTs were analyzed in the current study. Abscissa is the length of sequences in 50 bp intervals, whereas the total number of ESTs for each cluster is shown in the $\mathrm{Y}$-coordinate.

It is worth noticing that in the group of identified proteins, toxins account for $59.7 \%$ of the transcripts (30\% of the unique sequences). The distribution of all ESTs is depicted in Figure 2, it can be observed that ESTs coding for toxins are well represented in the $H$. gertschi venom gland transcriptome. Further, considering the non- matched ESTs as a possible source for new toxins, it can be assumed that these molecules are preferentially expressed over proteins related with the other cellular functions.

\section{GO-sorted annotated sequences}

All non-toxin nr-matched gene products were annotated in each of the three ontologies of GO: cellular component (CC), molecular function (MF) and biological processes (BP). Within each of these ontologies the categories with highest prevalence are: "intracellular" (11\% of total ESTs and $19 \%$ of unique sequences), "ribosome" (4.1\% and $7.4 \%)$, "mitochondrion" (4.1\% and $7.4 \%$ ) and "extracellular part" (6.1\% and 2.9\%) within CC; "catalytic activity" (16\% of total ESTs and unique sequences), "hydrolase activity" (10.9\% and 10.3\%), "protein binding" (4.8\% and $10.3 \%)$ and "ion binding" (7.5\% and 5.9\%) within $\mathrm{MF}$, and; "primary metabolic process" (7.5\% of total ESTs and $13.2 \%$ of unique sequences), "biosynthetic process" (4.8\% and $8.8 \%)$, "transport" $(4.1 \%$ and $8.8 \%)$ and "translation" (4.1\% and 7.4\%) within BP (Figure 3).

\section{Discussion}

Transcriptome analysis suggest cellular processes relevant for scorpion venom glands function

Although our sampling of the venom glands library is still incomplete, the diversity and nature of the annotated
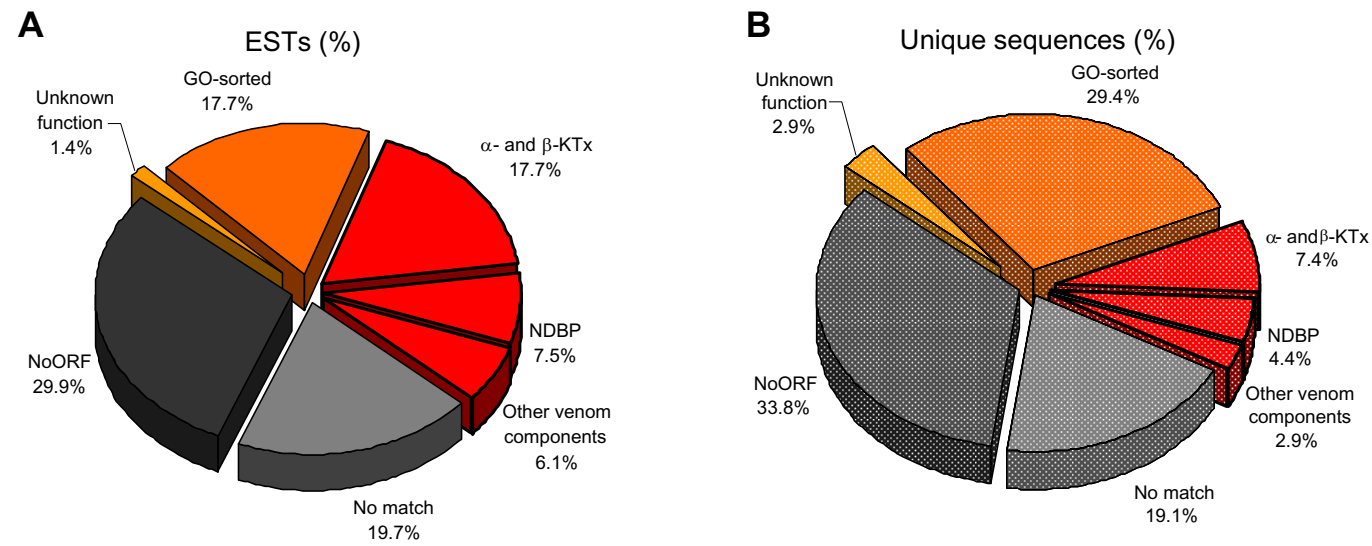

\section{Figure 2}

Relative proportion of each category of the transcripts from $\boldsymbol{H}$. gertschi venom gland library. A) Relative proportion of each category of the 147 total transcripts from $H$. gertschi venom gland. B) Relative proportion of the unique sequences (20 contigs and 48 singlets). "Unknown function" includes ESTs that presented identity with already described sequences with no functional assessment. "NoORF" includes sequences with non identified open reading frame. "No match" includes ESTs that did not match with currently known sequences. "GO-sorted" includes transcripts coding for proteins involved in cellular processes. " $\alpha$ and $\beta$-KTx" transcripts encode for putative $\mathrm{K}^{+}$toxins from $\alpha$ and $\beta$-families, respectively. "NDBP" comprises nondisulfide-bridged peptides. "Other venom components" includes both H. gertschi PLA2 and the Kunitz-type serine proteinase inhibitor. 


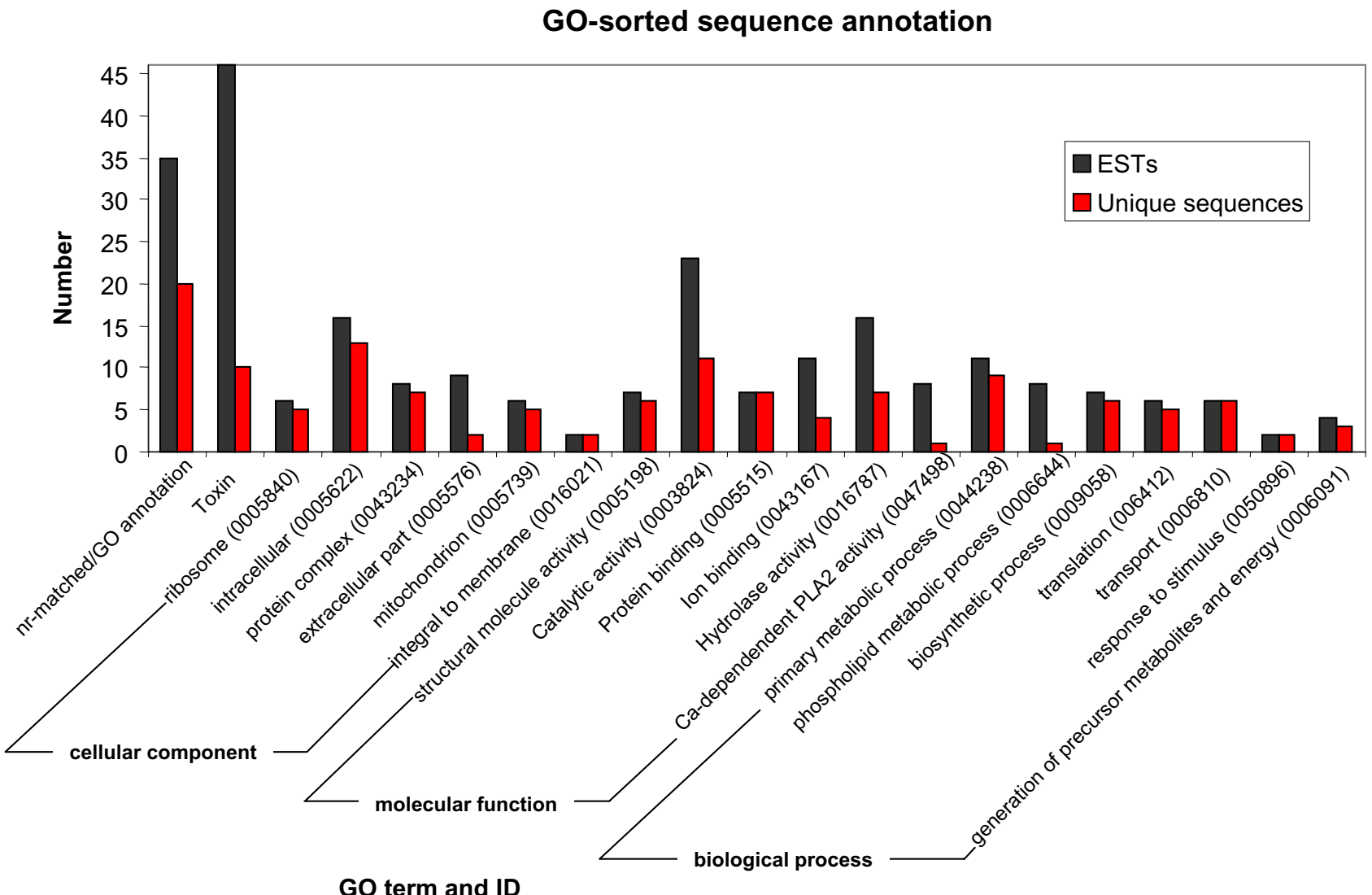

Figure 3

Gene Ontology-sorted sequence annotation. Functional classification of all nr-matched transcripts from the $H$. gertschi venom gland. The vertical axis shows the relative proportion of ESTs. The abscissa shows the categories within each of three ontologies: cellular component, molecular function and biological processes. For comparison, the relative proportion of toxinlike ESTs is also shown. All toxin-like sequences were assigned to the special set of the "biological process" ontology called "multi-organism process" (GO:005 I 704).

transcripts provide the first glimpse about molecular processes taking part in the scorpion venom gland cells. Since we constructed a non-amplified library, it could be expected that clone number reflects the actual prevalence of a given transcript. Moreover, by extension, different transcripts belonging to the same - confidentially assigned - GO category might suggest this category as important within the biological processes of scorpion venom glands.

For example, intuitively, the venom glands should support high protein synthesis and secretion in order to produce the large amounts of, secreted and renewable, venom proteins. In concordance, $8.2 \%$ of the total transcripts and $16.2 \%$ of the unique sequences match with either ribosomal components ( 1 contig and 4 singlets) or proteins involved in cellular trafficking ( 2 contigs and 1 singlet). Both processes are energetically costly and, consistently, $4.1 \%$ of whole ESTs and $7.4 \%$ of identified protein precursors match with components of the energyproducer organelle mitochondrion, whereas $2.7 \%$ and $4.4 \%$, respectively, are putative homologues of proteins directly involved in the energy-producer oxidative phosphorilation or tricarboxilyc acid cycle. Indeed, scorpions whose venoms were artificially depleted shows increased oxygen consumption [41].

The importance of correct protein processing in the context of scorpion venom gland is emphasized by the presence of transcripts encoding for proteins involved in correct folding (HGE008|2233), posttranslational processing (HGE014|2453 and HGE018|2232) or protea- 
some-dependent degradation of proteins (HGE010|2258). One of these (HGE014|2453), match with aminopeptidases of the M17 family (KOG2597), which are exopeptidases involved in the processing and regular turnover of intracellular proteins, although their precise role in cellular metabolism is unclear. In particular aminopeptidases of the M17 family cleave leucine residues from the N-terminal of polypeptide chains, but substantial rates are evident for all amino acids [42]. We predicted, by SignalP 3.0, its signal peptide-processing sequence (VAS-LK) suggesting that this transcript is secreted as a venom component, and indeed it could be important for posttranslational modifications of venomous gland components. Moreover, HGE018|2232 match with the glycosylating enzymes $\beta$-1,4-mannosyltransferases (KOG2941); which is consistent with the presence of glycosylated proteins in scorpion venoms (v.gr. [6]). transposon (nLTRrt) of clade R1 (sensu [43]). This nLTRrt clade is usually found inserted into telomeres and has been identified in several arthropods (including one arachnid) and some fungi [44]. It is worth noticing that mobile elements and their remnants account for large proportions of most eukaryotic genomes, in which they have had central roles in genome evolution and hypervariation. The expression of transposases indicates that mobile elements might contribute to the diversification of venom toxins. Recently, Glushkov et al., [45] reported the search for nLTRrt in 22 scorpion species, in which degenerate oligonucleotides based on consensus sequences of seven clades of nLTRrt were used to a PCR-based fishing approach. Unfortunately, even though these authors reported that PCR products of the expected size where obtained with R1-based degenerate primers, they only presented data for CR1, I and Jockey nLTRrt clades.

An interesting finding in our database was a transcript encoding the ribonuclease $\mathrm{H}$ domain of a non-LTR retro-

A

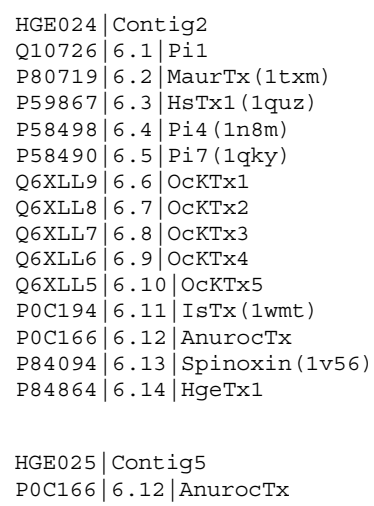

B

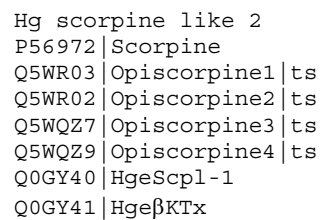

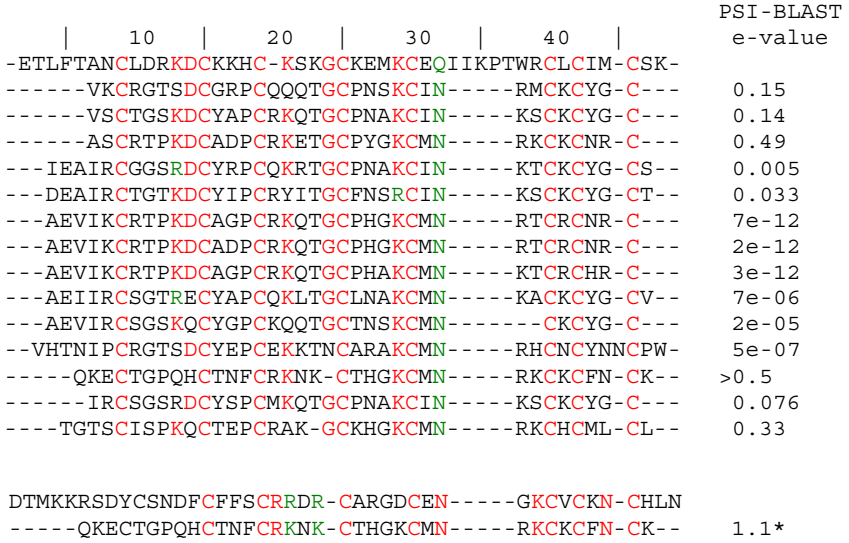

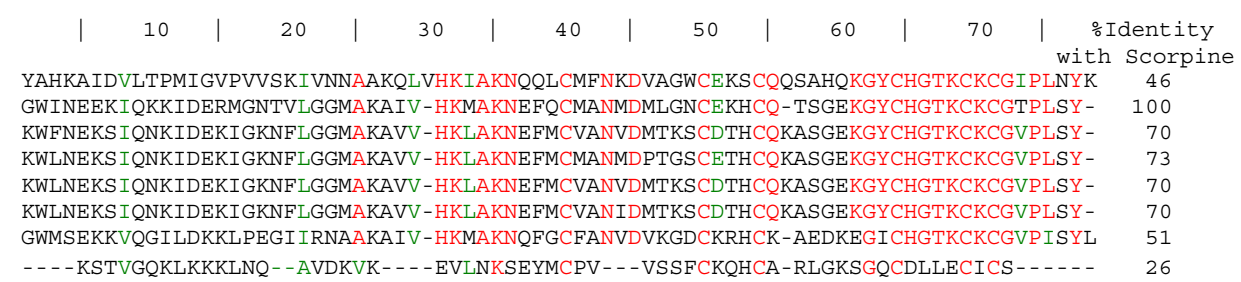

Figure 4

Scorpion toxin-like precursors in $H$. gertschi venom gland library. A) Predicted amino acid sequences of the potential $\alpha-K T x$. HGE024|Contig2 predicted sequence is aligned with all members of the $\alpha-K T x 6$ subfamily. HGE025|Contig5 is aligned with anuroctoxin ( $\alpha-K T \times 6.12$ ). PSI-BLAST e-values for the third iteration are shown. B) Predicted amino acid sequence of $\mathrm{Hg}$ scorpine like 2 and its alignment with others members of the scorpine-like group. The percentage of identity with scorpine is shown. See Supplementary Figure I for the complete nucleotide sequences of HGE024|Contig2, HGE025|Contig5 and Hg scorpine like 2. Each sequence starts with its SwissProt accession number followed by common names and Protein Data Bank codes between parentheses (where available). Systematic numbering (sensu [47,49]) for $\alpha-K T x$ is included between accession numbers and common names. Identical amino acids are in red colour and conserved ones in green. 


\section{Toxins and other venom components}

Our $H$. gertschi scorpion venom gland library is clearly enriched on toxin-like sequences, with more than $17 \%$ (in 4 contigs and 1 singlet) of the sequenced ESTs being similar to known families of scorpion toxins. Another $14 \%$ of the total ESTs (in 4 contigs) encodes for precursors which are homologues of previously characterized non-toxin scorpion venom components (see below). Considering that all these sequences contain putative signal peptides identified by SignalP 3.0 [46] - and their relative abundance, we suggest that these ESTs may encode secreted venom components. In fact, 3 of these clusters encode peptides already found in the venom of $H$. gertschi, which are currently being studied by our group [24 and unpublished].

\section{$\alpha-K T x s$}

Two clusters encoding potential $\alpha$-KTx peptides [47-49] were found and their translated sequences are shown in Figure 4 (and Supplementary Figure 1 in Additional file 1 ). One of those (HGE024|Contig2) is composed by 2 reads, and the other one (HGE025|Contig5) by 17 reads. Although the blastx search against public databases showed that HGE024|Contig2 sequence presents poor evalues $\left(>10^{-5}\right)$ with haemocyte defensins of insects and with some OcKTx's - predicted $\mathrm{K}^{+}$channel toxins from the scorpion Opistophthalmus carinatus [50], PSI-BLAST [51] with the translated sequence retrieved most of the members of the $\alpha$-KTx 6 subfamily (see [1,47-49]) within the first three iterations with good expectance values. Therefore, we propose that HGE024|Contig2 constitutes the precursor of a novel member of this subfamily of $\mathrm{K}^{+}$channel blockers. Similarly, the search of HGE025|Contig5 sequence against database revealed low similarity with anuroctoxin ( $\alpha$-KTx 6.12) from the scorpion Anuroctonus phaiodactylus, a high-affinity blocker of Kv1.3 channels of human T lymphocytes [52]. Again, the expectancy values were rather poor, but in this case, we were already able to purify the mature peptide from the venom of $H$. gertschi, and it is now under study. The prediction of its signal peptide and the $\mathrm{N}$-terminal amino acid sequence determined by automatic Edman degradation (data not shown) reveal that this toxin is in fact produced as a propeptide. This encode for a 67 amino acid-long peptide, containing three segments: an N-terminal signal peptide of 25 amino acid residues, a putative propeptide of 6 -amino acids and a mature peptide containing 36 residues (Supplementary Figure $1 \mathrm{~B}$ in Additional file 1 ). The mature peptide encoded by HGE025|Contig5 shows several conserved features of $\alpha$-KTx peptides, nonetheless its sequence is quite unique. We suspect that it might be the first member

A

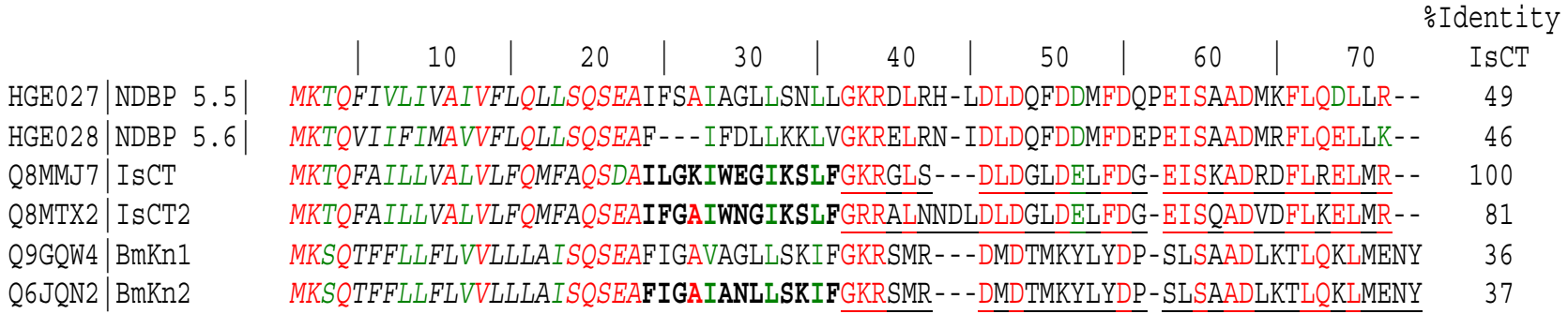

B

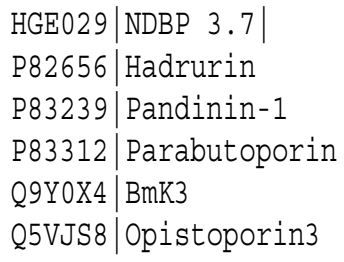

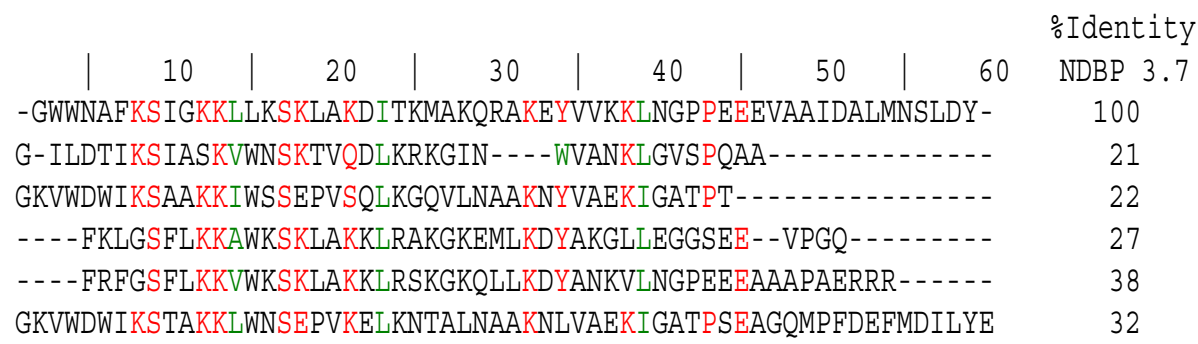

Figure 5

Predicted amino acid sequences of the novel non-disulfide-bridged peptides (NDBP). A) NDBP-5.5 and NDBP-5.6 are aligned with others scorpion cytolytic peptides; the percentage of identity with Is CT is shown. Putative signal peptides are in italics, whereas identified C-terminal prosequences and mature forms are underlined or in bold characters, respectively. B) Alignment of NDBP-3.7 with members of the NDBP 3 subfamily. See Supplementary Figure 2 for the complete nucleotide sequences encoding for NDBP-5.5, NDBP-5.6 and NDBP-3.7. Each sequence starts with its SwissProt accession number followed by common names. Identical amino acids are in red colour and conserved ones in green. 
of a new $\alpha$ KTx subfamily, but this remains to be clarified by ongoing analyses.

\section{$\beta-K T x$ and scorpine-like peptides}

One cluster ( 2 reads) coding the HgeßKTx and another (4 reads) coding the Hge scorpine like were identified in the transcriptome of $H$. gertschi and their sequence have already been reported [24]. Here we present a distinct EST, homologous to the scorpine-like group of long-chain three disulphide-bridged scorpion venom peptides, named Hge scorpine like 2 (Figure $4 \mathrm{~b}$ and Supplementary Figure 1C in Additional file 1).

\section{Cytolytic peptides}

Two clusters encoding IsCT-like precursors were found in $H$. gertschi transcriptome. IsCT and IsCT2 are antimicrobial linear peptides isolated from the scorpion Opisthacanthus madagascariensis [53]. They possess broad activity spectra against Gram positive and negative bacteria as well as fungi and relatively weak haemolytic activity against sheep red blood cells. Additionally to the signal peptide, their precursors contain an uncommon acidic propeptide at the C-terminal (Supplementary Figure 2A in Additional file 1). Figure $5 \mathrm{a}$ shows both IsCT-like translated sequences, the more abundant comprising 7 reads and the other one represented by 2 reads, classified as nondisulfide-bridged peptides (NDBP) NDBP-5.5 and NDBP5.6, respectively (following the nomenclature rules of [7]).

\section{Bradykinin-potentiating peptide like}

One cluster (HGE029|NDBP_3.7, 2 reads; see Supplementary Figure 2B in Additional file 1) encodes a homo- logue of the bradykinin-potentiating peptide precursor (BmK3 or BmKpbb) from the scorpion Mesobuthus martensii, classified as NDBP-3.3 [7]. The angiotensin-bradykinin system is a central hormonal system for the regulation of blood pressure. The angiotensin-converting enzyme (ACE) converts angiotensin I to angiotensin II and degrades bradykinin. Bradykinin potentiating peptides have been isolated from Tityus serrulatus (peptide $\mathrm{T}$ [54]) and Buthus occitanus (K12 [55]). Peptide T, a 13amino acid linear peptide, potentiates the contractile activity of bradykinin on isolated smooth muscle, inhibits the hydrolysis of bradykinin by ACE, and enhances the depressor effect of bradykinin on arterial blood pressure in the anesthetized rats [54]. Peptide K12 displays similar bradykinin potentiating activities [55]. BmKbpp was identified from $B$. martensii Karsch by cDNA cloning based on the peptide $\mathrm{K} 12$ amino acid sequence [56]. The last 21 residues of C-terminal region of BmKbpp showed 57\% similarity with peptide K12. Based on the fact that BmKbpp also exhibits high similarity with parabutoporin and others antimicrobial peptides from scorpions, it was suggested that BmKbpp may be a molecule with a dualfunction, and that the BmKbpp precursor may be processed in two alternative ways to produces two different mature molecules: BmKbpp and a peptide with only the C-terminal 21 residues of BmKbpp [7]. Figure 5b shows the bradykinin-potentiating peptide like from $H$. gertschi, here named NDBP-3.7.

\section{Phospholipases}

A cluster ( 8 reads) of a new homologue of scorpion venom phospholipases A2 (ScpPLA2) was identified in the $H$. gertschi library (see Supplementary Figure 3 in
HGE031 PLA2 Hadge P59888 Imparatoxin I Q6PXP0|Phospholipin Q3YAU5|PLA2_Hetfu|ts Q6T178|PLA2_Mesta|ts

HGE031|PLA2_Hadge P59888|Imparatoxin I Q6PXP0|Phospholipin Q3YAU5|PLA2_Hetfu|ts Q6T178|PLA2_Mesta|ts

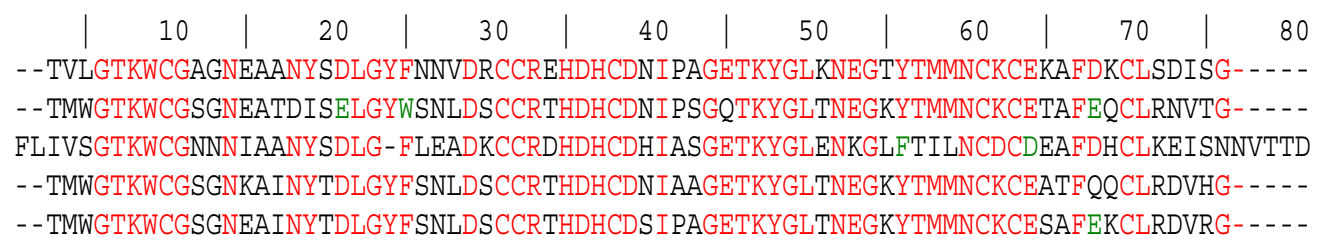

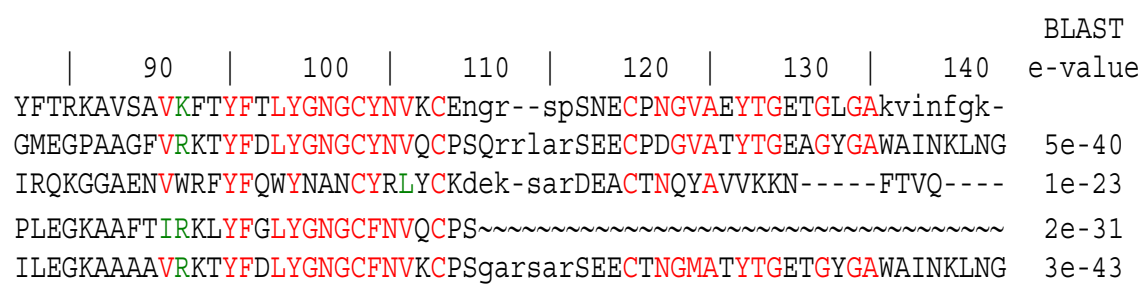

\section{Figure 6}

Putative mature sequence of phospholipase A2 precursor. Predicted amino acid sequence of $H$. gertschi PLA2 (HGE03 I|PLA2) aligned with other scorpion venom PLA2. BLAST e-values are shown. See Supplementary Figure 3 for the nucleotide sequence of HGE03 I|PLA2. Each sequence starts with its SwissProt accession number followed by common names. Identical amino acids are in red colour and conserved ones in green. 


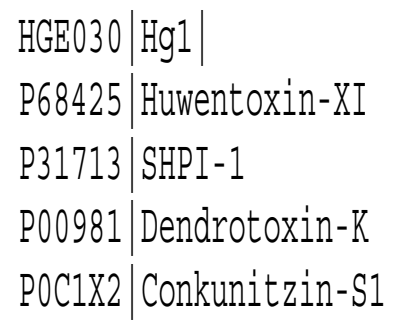

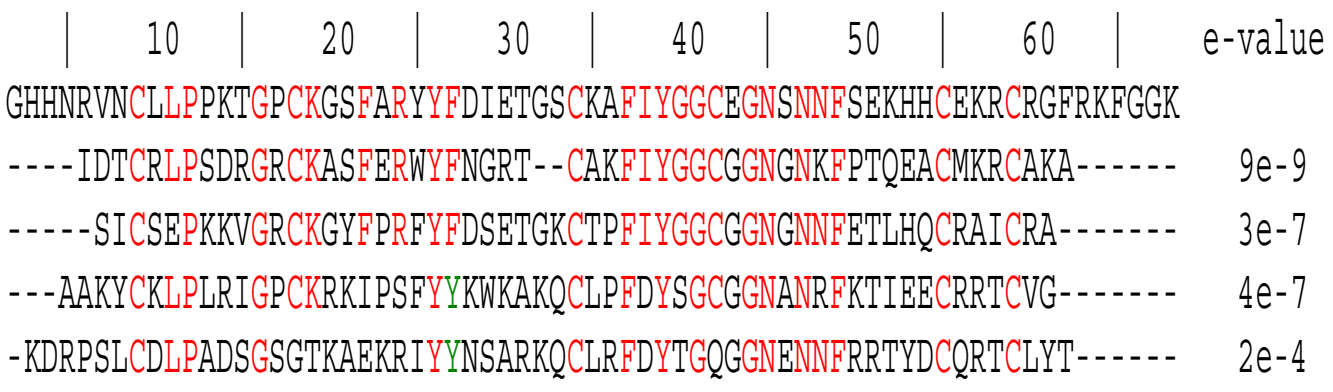

\section{Figure 7}

Multiple sequence alignment of the KU-type proteins of venomous organisms. Predicted amino acid sequence of HGE030|HgI aligned with other venom-derived members of the Kunitz-type serine proteinase inhibitors. BLAST e-values with P68425 (spider Ornithoctonus huwena), P3I7I 3 (sea anemone Stichodactyla helianthus), P0098I (snake Dendroaspis polylepis polylepis) and P0CIX2 (cone snail Conus striatus) are shown. See Supplementary Figure 4 for the complete nucleotide sequence of HGE030|HgI. Each sequence starts with its SwissProt accession number followed by common names. Identical amino acids are in red colour and conserved ones in green.

Additional file 1). The mature form of ScpPLA2 are composed by two subunits, the large ones consisting of approximately 105 amino acid residues, whereas the small subunits have between 18 and 27 residues; their heterodimeric form is stabilized by one interchain disulphide bridge $[5,6]$. The ScpPLA2 are expressed from a single message, from which the N-terminal propeptide, a penta or hexapeptide internal segment and a short C-terminal region are excised to give the heterodimeric mature form of the enzyme. In Figure 6, these regions are identified on the sequence of the predicted $H$. gertschi PLA2; the assignment was based on multiple sequence alignment of known ScpPLA2. PLA2s are enzymes that catalyze the hydrolysis of the $s n-2$ acyl bonds of $s n-3$ phospholipids, and are normal cellular mediators involved in different responses, such as inflammation, blood hemostasis and others. Many animal venoms posses PLA2s that mediate several toxic responses, like cytotoxicity, neurotoxicity, myotoxicity, edema and blood coagulation disturbs. Based on their primary structure, these toxins can be classified in tree distinct classes: class I is found in Elapidae snakes venom; class II is found in the Viperidae family of snakes; and class III that was identified for the first time in the bee venom. Latter they were found in other invertebrates such as jellyfish, marine snails, and scorpion venoms, but they are also present in vertebrates, like the venomous lizard Heloderma [57].

\section{Kunitz-type carboxypeptidase inhibitor}

One EST (HGE030|Hg1 (Supplementary Figure 4 in Additional file 1), is homologous to KOG4295, which contains serine proteinase inhibitors of the Kunitz type (KU family). Proteins with KU have been identified in several venomous organisms, like snakes [58], sea anemones
[59], cone snails [60] and spiders [61]. However, this is the first report of a KU-type precursor in scorpions. Figure 7 shows the multiple sequence alignment of HGE030|Hg1 with other KU-type venom components. Although the precise role of HGE030|Hg1 in the context of scorpion venom remains to be determined - whether it display neurotoxic or proteinase inhibitor activity -, the ubiquitous presence of proteinase inhibitors suggest a common trend in venomous organisms, deserving further studies.

\section{Conclusion}

Gene cloning of animal toxins has been extensively performed by PCR method, using primers deduced from direct protein sequencing, usually by Edman degradation or mass spectrometry analysis. These studies are aimed at the isolation of specific active components. However, this approach is not entirely suitable for search of unforeseen components that could be present in the venomous gland under study. The strategy is biased by the fact that only those genes that are sharing sequence similarities are usually discovered by this technique. For this reason, we adopted the molecular approach of generating and analyzing ESTs from the $H$. gertschi venom gland as the strategy to produce a general overview of the venom gland transcriptome. This strategy confirms the highly specialized nature of scorpion venom glands as toxin-producer, allowing the description, for the first time, of putative proteins that certainly are involved in cellular processes relevant for the venom glands' function. Additionally, the unguided mining also reveals novel predicted venom components, highlighting the usefulness of the transcriptome approach to improve venom profiling. 


\section{Methods \\ cDNA library construction}

A cDNA library was constructed from total RNA extracted from a single telson of a $H$. gertschi scorpion. The scorpion was milked 5 days before RNA extraction. For RNA isolation the 'Total RNA Isolation System' of Promega (Madison, WI) was used. With this material a full-length cDNA phagemid library was prepared using the SMART cDNA Library Construction Kit (CLONTECH Lab., Palo Alto, $\mathrm{CA})$. The titre of the non-amplified cDNA library obtained was $2.8 \times 10^{5} \mathrm{cfu} / \mathrm{mL}$ with $99 \%$ recombinant clones. For the PCR the oligonucleotides TriplEx2-5' (from CLONTECH Lab, Palo Alto, CA) and CD3/3' (5'-AAT CTA GAG GCC GAG GCG GCC GAC ATG-3') [24], designed on the basis of the CDSIII/3' sequence tag used for library construction, were used as primers.

\section{DNA sequencing and bioinformatic analyses}

Selected plasmids were isolated according to a standard alkaline lysis protocol, and single-pass sequencing of the 5'-termini was conducted with the primer TriplEx2-5' (CLONTECH Lab, Palo Alto, CA) using an automatic machine (Model 3100, Applied Biosystems, Foster city, CA) according to the manufacturer's instructions. The nucleotide sequences obtained in this work are deposited in dbEST (GenBank: EL698878-EL698945). To extract the high quality sequence region, the ESTs were subjected to the Phred program [62] with the window length set to 100 and the standard quality to 20. The CrossMatch program was used to remove vector and $E$. coli DNA sequences. ESTs that shared an identity of $>95$ out of 100 nucleotides were assembled in contiguous sequences with the CAP3 program [63]. All these bioinformatics analysis were simultaneously run at [64] using default setup. $H$. gertschi venom gland ESTs (clusters and singlets) were searched against nr database [65] using blastx and blastn algorithms [66] with an e-value cutoff set to $<10^{-5}$ to identify putative functions of the new ESTs. The signal peptide was predicted with the SignalP 3.0 program [46]. Multiple sequences alignments were obtained using T-COFFEE [67] or CLUSTAL_X [68]. The pairwise identities were calculated with BioEdit [69]. In order to evaluate the most expressed ESTs, a less stringent alignment using a shared identity of $>65$ out of 100 nucleotides was used.

With the aim of providing more useful sequence annotations for comparative studies, we selected to identify the eukaryotic orthologous group [38] to which each sequence would belong, instead of reporting the - most often used - highest scored BLAST hit. The best match for each sequence with KOG database was identified by Kognitor - a BLAST-based web server that search within KOGs only - and validated by independent PSI-BLAST searches [51]. The rationale followed was that PSI-BLAST should return all members of the given KOG within the first two iterations. Furthermore, in order to improve sequence annotation and to gain insights into the cellular processes where each sequence could be involved, the ESTs were submitted to GOblet [40] and AmiGO [39] web servers. The aim of this procedure was to identify relevant Gene Ontology terms. Both programs perform blastx searches against UniProt (GOblet) and all GO-annotated protein databases (AmiGO). Cross-validation with identified KOGs was performed by inspection of the retrieved hits in order to identify homologues of the corresponding KOGs. Subsequently the GenBank entries of the given KOG were used as query for AmiGO. GO terms were assigned only when the novel sequence and at least one sequence of each KOGs retrieved coincident hits (usually five or more out of the ten highest-scoring hits).

\section{Authors' contributions}

EFS and EDG designed and carried out most of the experiments described, with some technical assistance (see acknowledgements). EFS and RCRV analyzed the data and wrote the initial draft of the manuscript. LDP supervised the entire project and revised the final version of the manuscript. All authors read and approved the manuscript.

\section{Additional material}

\section{Additional File 1 \\ Supplementary figures. Four figures in a PDF document, including nucle- otide and translated sequences of putative venom components. Click here for file \\ [http://www.biomedcentral.com/content/supplementary/1471- 2164-8-119-S1.pdf] \\ Additional File 2 \\ dbEST submission. Complete submission to the dbEST database in raw text format. Each entry includes: cluster size and, where applicable, assigned eukaryotic orthologous group and relevant Gene Ontology terms. Click here for file \\ [http://www.biomedcentral.com/content/supplementary/1471- 2164-8-119-S2.txt]}

\section{Acknowledgements}

The authors are indebted to Dr Blanca I. García and M.Sc. Timoteo Olamendi-Portugal for their technical assistance. This work was partially financed by grants from CNPq - Brazil number 201294/2003-6 (to EFS), CONACyT-Mexico and CNPq-Brazil (to LDP and EFS) and grants from Dirección General de Asuntos del Personal Académico (Universidad Nacional Autónoma de México) number IN227507 and CONACyT-Fondo Salud number 14109 (Mexican Government) to LDP.

\section{References}

I. Jungo $F$, Bairoch $A$ : Tox-Prot, the toxin protein annotation program of the Swiss-Prot protein knowledgebase. Toxicon 2005, 45:293-301.

2. Tan PT, Veeramani A, Srinivasan KN, Ranganathan S, Brusic V: SCORPION2: a database for structure-function analysis of scorpion toxins. Toxicon 2006, 47:356-363. 
3. Possani LD, Rodríguez de la Vega RC: Scorpion venom peptides. In Handbook of Biologically Active Peptides Edited by: Kastin AJ. San Diego, Academic Press; 2006:339-354.

4. Ramanaiah M, Parthasarathy PR, Venkaiah B: Purification and properties of phospholipase A2 from the venom of scorpion, (Heterometrus fulvipes). Biochem Int 1990, 20:931-940.

5. Zamudio FZ, Conde R, Arevalo C, Becerril B, Martin BM, Valdivia HH Possani LD: The mechanism of inhibition of ryanodine receptor channels by imperatoxin I, a heterodimeric protein from the scorpion Pandinus imperator. J Biol Chem 1997, 272: II886-II894

6. Valdez-Cruz NA, Batista CV, Possani LD: Phaiodactylipin, a glycosylated heterodimeric phospholipase $A$ from the venom of the scorpion Anuroctonus phaiodactylus. Eur J Biochem 2004, 27I: | 453-| 464.

7. Zeng XC, Corzo G, Hahin R: Scorpion venom peptides without disulfide bridges. IUBMB Life 2005, 57:|3-2I.

8. Luo F, Zeng XC, Hahin R, Cao ZJ, Liu H, Li WX: Genomic organization of four novel nondisulfide-bridged peptides from scorpion Mesobuthus martensii Karsch: gaining insight into evolutionary mechanism. Peptides 2005, 26:2427-2433.

9. Pimenta AM, Stocklin R, Favreau P, Bougis PE, Martin-Eauclaire MF: Moving pieces in a proteomic puzzle: mass fingerprinting of toxic fractions from the venom of Tityus serrulatus (Scorpiones, Buthidae). Rapid Commun Mass Spectrom 2001, I 5: I562-I572

10. Batista CV, del Pozo L, Zamudio FZ, Contreras S, Becerril B, Wanke E, Possani LD: Proteomics of the venom from the Amazonian scorpion Tityus cambridgei and the role of prolines on mass spectrometry analysis of toxins. J Chromatogr B 2004, 803:55-66.

II. Diego-Garcia E, Batista CV, Garcia-Gomez BI, Lucas S, Candido DM, Gomez-Lagunas F, Possani LD: The Brazilian scorpion Tityus costatus Karsch: genes, peptides and function. Toxicon 2005 45:273-283.

12. Barona J, Batista CV, Zamudio FZ, Gomez-Lagunas F, Wanke E, Otero R, Possani LD: Proteomic analysis of the venom and characterization of toxins specific for $\mathrm{Na}+-$ and $\mathrm{K}+$-channels from the Colombian scorpion Tityus pachyurus. Biochim Biophys Acta 2006, 1764:76-84.

13. Batista CV, D'Suze G, Gomez-Lagunas F, Zamudio FZ, Encarnacion S, Sevcik C, Possani LD: Proteomic analysis of Tityus discrepans scorpion venom and amino acid sequence of novel toxins. Proteomics 2006, 6:37|8-3727.

14. Borges A, Garcia CC, Lugo E, Alfonzo MJ, Jowers MJ, Op den Camp $\mathrm{H}$ ): Diversity of long-chain toxins in Tityus zulianus and Tityus discrepans venoms (Scorpiones, Buthidae): molecular, immunological, and mass spectral analyses. Comp Biochem Physiol C 2006, I 42:240-252.

15. Caliskan F, Garcia BI, Coronas FI, Batista CV, Zamudio FZ, Possani LD: Characterization of venom components from the scorpion Androctonus crassicauda of Turkey: peptides and genes. Toxicon 2006, 48: 12-22.

16. Nascimento DG, Rates B, Santos DM, Verano-Braga T, Barbosa-Silva A, Dutra AA, Biondi I, Martin-Eauclaire MF, De Lima ME, Pimenta AM Moving pieces in a taxonomic puzzle: venom 2D-LC/MS and data clustering analyses to infer phylogenetic relationships in some scorpions from the Buthidae family (Scorpiones). Toxicon 2006, 47:628-639.

17. Possani LD, Becerril B, Delepierre M, Tytgat J: Scorpion toxins specific for Nat-channels. Eur J Biochem 1999, 264:287-300.

18. Goudet C, Chi CW, Tytgat J: An overview of toxins and genes from the venom of the Asian scorpion Buthus martensi Karsch. Toxicon 1999.

19. Zeng XC, Wang SX, Zhu Y, Zhu SY, Li WX: Identification and functional characterization of novel scorpion venom peptides with no disulfide bridge from Buthus martensii Karsch. Peptides 2004, 25: I 43-150.

20. Zeng XC, Luo F, Li WX: Molecular dissection of venom from Chinese scorpion Mesobuthus martensii: identification and characterization of four novel disulfide-bridged venom peptides. Peptides 2006, 27: $1745-1754$.

21. Soleglad ME, Fet V: High-level systematics and phylogeny of the extant scorpions (Scorpiones: Orthosterni). Euscorpius 2003:11.
22. Torres-Larios A, Gurrola GB, Zamudio FZ, Possani LD: Hadrurin, a new antimicrobial peptide from the venom of the scorpion Hadrurus aztecus. Eur J Biochem 2000, 267:5023-3I.

23. Schwartz EF, Schwartz CA, Gomez-Lagunas F, Zamudio FZ, Possani LD: HgeTxI, the first K+-channel specific toxin characterized from the venom of the scorpion Hadrurus gertschi Soleglad. Toxicon 2006, 48: 1046-1053.

24. Diego-Garcia E, Schwartz EF, D'Suze G, Gonzalez SA, Batista CV, Garcia BI, Rodríguez de la Vega RC, Possani LD: Wide phylogenetic distribution of Scorpine and long-chain beta-KTx-like peptides in scorpion venoms: identification of "orphan" components. Peptides 2007, 28:3I-37.

25. Whitton C, Daub J, Thompson M, Blaxter M: Expressed sequence tags: medium-throughput protocols. Methods Mol Biol 2004, 270:75-92.

26. Yang Y, Cun S, Xie X, Lin J, Wei J, Yang W, Mou C, Yu C, Ye L, Lu Y, Fu Z, Xu A: EST analysis of gene expression in the tentacle of Cyanea capillata. FEBS Lett 2003, 538:|83-19|.

27. Hydra EST database [http://www.hydrabase.org]

28. Pi C, Liu J, Peng C, Liu Y, Jiang X, Zhao Y, Tang S, Wang L, Dong M, Chen S, Xu A: Diversity and evolution of conotoxins based on gene expression profiling of Conus litteratus. Genomics 2006, 88:809-819.

29. Magalhaes GS, Junqueira-de-Azevedo ILM, Lopes-Ferreira M, Lornzini DM, Ho PL, Moura-da-Silva AM: Transcriptome analysis of expressed sequence tags from the venom glands of the fish Thalassophryne nattereri. Biochimie 2006, 88:693-699.

30. Cidade DA, Simao TA, Davila AM, Wagner G, Junqueira-de-Azevedo Ide L, Ho PL, Bon C, Zingali RB, Albano RM: Bothrops jararaca venom gland transcriptome: analysis of the gene expression pattern. Toxicon 2006, 48:437-46I.

31. Wagstaff SC, Harrison RA: Venom gland EST analysis of the saw-scaled viper, Echis ocellatus, reveals novel alpha9betal integrin-binding motifs in venom metalloproteinases and a new group of putative toxins, renin-like aspartic proteases. Gene 2006, 377:2 I-32.

32. Zhang B, Liu Q, Yin W, Zhang $X$, Huang $Y$, Luo $Y$, Qiu $P$, Su $X, Y u J$, Hu S, Yan G: Transcriptome analysis of Deinagkistrodon acutus venomous gland focusing on cellular structure and functional aspects using expressed sequence tags. BMC Genomics 2006 , 7:152.

33. Kozlov S, Malyavka A, McCutchen B, Lu A, Schepers E, Herrmann R, Grishin $\mathrm{E}$ : A novel strategy for the identification of toxinlike structures in spider venom. Proteins 2005, 59:131-140.

34. Audic S, Claverie J-M: The significance of digital gene expression profiles. Genome Res 1997, 7:986-995

35. Dana AN, Hillenmeyer ME, Lobo NF, Kern MK, Romans PA, Collins $\mathrm{FH}$ : Differential gene expression in abdomens of the malaria vector mosquito, Anopheles gambiae, after sugar feeding, blood feeding and Plasmodium berghei infection. BMC Genomics 7:119.

36. Schmitt AO, Specht T, Beckmann G, Dahl E, Pilarsky CP, Hinzmann $B$, Rosenthal A: Exhaustive mining of EST libraries for genes differentially expressed in normal and tumour tissues. Nucleic Acids Res 1999, 27:4251-4260.

37. Liu D, Graber JH: Quantitative comparison of EST libraries requires compensation for systematic biases in cDNA generation. BMC Genomics 2006, 7:77.

38. Tatusov RL, Fedorova ND, Jackson JW, Jacobs AR, Kiryutin B, Koonin EV, Krylov DM, Mazumder R, Mekhedov SL, Nikolskaya AN, Rao BS, Smirnov S, Sverdlov AV, Vasudevan S, Wolf YI, Yin J], Natale DA: The COG database: an updated version includes eukaryotes. BMC Bioinformatics 2003, 4:4I.

39. The Gene Ontology Consortium: Gene Ontology: tool for the unification of biology. Nat Genet 2000, 25:25-29.

40. Groth D, Lehrach H, Hennig S: GOblet: a platform for Gene Ontology annotation of anonymous sequence data. Nucleic Acids Res 2004, 32:W313-W319.

4I. Nisani Z, Dunbar SG, Hayes WK: Cost of venom regeneration in Parabuthus transvaalicus (Arachnida: Buthidae). Comp Biochem Physiol A 2007, 147:509-5 I3.

42. Matsui $M$, Fowler JH, Walling LL: Leucine aminopeptidases: diversity in structure and function. Biol Chem 2006, 387: $1535-1544$. 
43. Malik HS, Burke WD, Eickbush TH: The age and evolution of nonLTR retrotransposable elements. Mol Biol Evol 1999, 16:793-805

44. Malik HS: Ribonuclease $\mathbf{H}$ evolution in retrotransposable elements. Cytogenet Genome Res 2005, I I 0:392-40 I.

45. Glushkov S, Novikova O, Blinov A, Fet V: Divergent non-LTR retrotransposon lineages from the genomes of scorpions (Arachnida: Scorpiones). Mol Genet Genomics 2006, 275:88-296.

46. SignalP 3.0 Server [http://www.cbs.dtu.dk/services/SignalP/]

47. Tytgat J, Chandy KG, Garcia ML, Gutman GA, Martin-Eauclaire MF, van der Walt JJ, Possani LD: A unified nomenclature for shortchain peptides isolated from scorpion venoms: alpha-KTx molecular subfamilies. Trends Pharmacol Sci 1999, 20:444-447.

48. Rodríguez de la Vega RC, Possani LD: Current views on scorpion toxins specific for K+-channels. Toxicon 2004, 43:865-875.

49. UniProt Consortium: The Universal Protein Resource (UniProt). Nucleic Acids Res 2007, 35:D 193-197.

50. Zhu S, Huys I, Dyason K, Verdonck F, Tytgat J: Evolutionary trace analysis of scorpion toxins specific for K-channels. Proteins 2004, 54:36I-370.

5I. Altschul SF, Madden TL, Schaffer AA, Zhang J, Zhang Z, Miller W, Lipman 50DJ: Gapped BLAST and PSI-BLAST: a new generation of protein database search programs. Nucleic Acids Res 1997 25:3389-3402.

52. Bagdany M, Batista CV, Valdez-Cruz NA, Somodi S, Rodríguez de la Vega RC, Licea AF, Varga Z, Gaspar R, Possani LD, Panyi G: Anuroctoxin, a new scorpion toxin of the alpha-KTx 6 subfamily, is highly selective for KvI.3 over IKCaI ion channels of human T lymphocytes. Mol Pharmacol 2005, 67: 1034-1044.

53. Dai L, Corzo G, Naoki H, Andriantsiferana M, Nakajima T: Purification, structure-function analysis, and molecular characterization of novel linear peptides from scorpion Opisthacanthus madagascariensis. Biochem Biophys Res Commun 2002, 293: $1514-1522$.

54. Ferreira LA, Alves EW, Henriques OB: Peptide T, a novel bradykinin potentiator isolated from Tityus serrulatus scorpion venom. Toxicon 1993, 31:94|-947.

55. Meki AR, Nassar AY, Rochat H: A bradykinin-potentiating peptide (peptide $\mathrm{K} / 2$ ) isolated from the venom of Egyptian scorpion Buthus occitanus. Peptides 1995, 16: | 359-1365.

56. Zeng XC, Li WX, Peng F, Zhu ZH: Cloning and characterization of a novel cDNA sequence encoding the precursor of a nove venom peptide (BmKbpp) related to a bradykinin-potentiating peptide from Chinese scorpion Buthus martensii Karsch. IUBMB Life 2000, 49:207-2I0.

57. Valdez-Cruz NA, Segovia L, Corona M, Possani LD: Sequence analysis and phylogenetic relationships of gene encoding heterodimeric Phospholipases A2 from the venom of the scorpion Anuroctonus phaiodactylus. Gene 2007, 396: I49-I 58.

58. Harvey AL, Robertson B: Dendrotoxins: structure-activity relationships and effects on potassium ion channels. Curr Med Chem 2004, I I:3065-3072.

59. Bruhn HD, Beress L: Polyvalent proteinase inhibitor from the sea anemone Anemonia sulcata: effect on coagulation and fibrinolysis. Thromb Haemost 1978, 39:552-554.

60. Bayrhuber M, Vijayan V, Ferber M, Graf R, Korukottu J, Imperial J, Garrett JE, Olivera BM, Terlau H, Zweckstetter M, Becker S: Conkunitzin-SI is the first member of a new Kunitz-type neurotoxin family. Structural and functional characterization. J Bio Chem 2005, 280:23766-23770.

61. Peng K, Lin Y, Liang SP: Nuclear magnetic resonance studies on huwentoxin-XI from the Chinese bird spider Ornithoctonus huwena: I $5 \mathrm{~N}$ labeling and sequence-specific I H, I5N nuclear magnetic resonance assignments. Acta Biochim Biophys Sin (Shanghai) 2006, 38:457-466.

62. Lazo GR, Tong J, Miller R, Hsia C, Rausch CY, Kang Y, Anderson OD: Software scripts for quality checking of high throughput nucleic acid sequencers. Biotechniques 200I, 30:1300-1305.

63. Huang X, Madan A: CAP3: a DNA sequence assembly program. Genome Res 1999, 9:868-877.

64. Laboratório de Biologia Molecular - Universidade de Brasilia [https://www.biomol.unb.br/]

65. NCBI BLAST [http://www.ncbi.nlm.nih.gov/blast]

66. Altschul SF, Gish W, Miller W, Myers EW, Lipman DJ: Basic local alignment search tool. I Mol Biol 1990, 21 5:403-4I0.
67. Notredame C, Higgins DG, Heringa J: T-Coffee: a novel method for fast and accurate multiple sequence alignment. J Mol Biol 2000, 302:205-217.

68. Thompson JD, Gibson TJ, Plewniak F, Jeanmougin F, Higgins DG: The CLUSTAL_X windows interface: flexible strategies for multiple sequence alignment aided by quality analysis tools. Nucleic Acids Res 1997, 25:4876-4882.

69. BioEdit [http://www.mbio.ncsu.edu/BioEdit/bioedit.html]
Publish with Biomed Central and every scientist can read your work free of charge

"BioMed Central will be the most significant development for disseminating the results of biomedical research in our lifetime. "

Sir Paul Nurse, Cancer Research UK

Your research papers will be:

- available free of charge to the entire biomedical community

- peer reviewed and published immediately upon acceptance

- cited in PubMed and archived on PubMed Central

- yours - you keep the copyright
BioMedcentral 